\title{
Efficacy of Amlodipine/Olmesartan \pm Hydrochlorothiazide in Patients Uncontrolled on Prior Calcium Channel Blocker or Angiotensin II Receptor Blocker Monotherapy
}

Joel Neutel $\cdot$ Ali Shojaee $\cdot$ Jen-Fue Maa

To view enhanced content go to www.advancesintherapy.com Received: May 7, 2012 / Published online: July 4, 2012

(c) The Author(s) 2012. This article is published with open access at Springerlink.com

\section{ABSTRACT}

Introduction: While monotherapy is often recommended as initial treatment, most patients require dose escalation and add-on agents to achieve their blood pressure (BP) goal. This secondary analysis evaluated the efficacy and safety of initiating patients on a regimen of fixeddose amlodipine (AML)/olmesartan medoxomil $(\mathrm{OM}) \pm$ hydrochlorothiazide $(\mathrm{HCTZ})$ who were uncontrolled on prior monotherapy with a calcium channel blocker (CCB) or angiotensin II receptor blocker (ARB).

Methods: Patients uncontrolled on prior monotherapy with CCB or ARB therapy were

J. Neutel $(\bowtie)$

Orange County Research Center, Department of Clinical Pharmacology, 14351 Myford Road, Tustin, CA 92780, USA

e-mail: jmneutel@aol.com

A. Shojaee · J-F. Maa

Daiichi Sankyo, Inc., Parsippany, NJ, USA

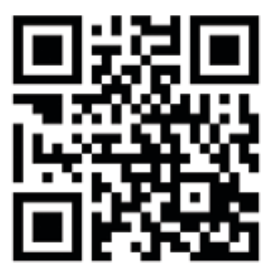

Enhanced content for Advances in Therapy articles is available on the journal web site: www.advancesintherapy.com initiated on AML/OM 5/20 mg and up-titrated every 4 weeks to AML/OM 5/40 mg, AML/OM 10/40 mg, AML/OM 10/40 + HCTZ $12.5 \mathrm{mg}$, and AML/OM 10/40 + HCTZ 25 mg. Patients were up-titrated to a higher AML/OM dose if mean seated cuff BP (SeBP) was $\geq 120 / 70 \mathrm{mmHg}$, and up-titrated to any HCTZ dose if mean SeBP was $\geq 125 / 75 \mathrm{mmHg}$. The primary efficacy endpoint was the cumulative proportion of patients achieving a seated cuff systolic BP (SeSBP) goal of $<140 \mathrm{mmHg}$ (<130 mmHg for patients with diabetes) after 12 weeks. Secondary endpoints included mean change from baseline in SeBP and ambulatory BP, ambulatory BP target achievement, and safety.

Results: For the prior ССВ ( $n=118$; baseline SeBP: $153.4 / 91.5 \mathrm{mmHg})$ and ARB $(n=237$; 154.6/92.6 mmHg) groups, SeSBP goal achievement after 12 weeks was $72.7 \%$ and $76.9 \%$, respectively. Mean changes ( \pm SE) from baseline in SeBP were dose proportional for prior $\mathrm{CCB}$ and $\mathrm{ARB}$ patients, ranging from $-9.9( \pm 1.25) /-5.8( \pm 0.83) \mathrm{mmHg}$ and $-13.9( \pm 0.79) /-7.6( \pm 0.47) \mathrm{mmHg}$ at the AML/OM 5/20 mg dose, respectively, to -21.8 $( \pm 1.68) /-11.6( \pm 1.12) \mathrm{mmHg}$ and -26.2 $( \pm 1.31) /-15.0( \pm 0.86) \mathrm{mmHg}$ at the AML/OM $10 / 40 \mathrm{mg}+$ HCTZ $25 \mathrm{mg}$ dose $(P<0.0001$ for all). 
Conclusion: An AML/OM-based titration regimen was efficacious in achieving BP goal in patients uncontrolled on prior monotherapy with a CCB or ARB.

Keywords: Amlodipine; Angiotensin II receptor blocker; Blood pressure; Calcium channel blocker; Combination therapy; Hydrochlorothiazide; Hypertension; Olmesartan medoxomil

\section{INTRODUCTION}

Hypertension is a prevalent condition that if left uncontrolled can increase cardiovascular morbidity and mortality as manifested by increased incidence of ischemic heart disease and stroke [1]. For many patients, achievement of blood pressure (BP) control requires escalation of monotherapy to combination therapy with agents from multiple pharmacological classes [2, 3]. Despite the recommendations of clinical practice guidelines, only $48.4 \%$ of patients have their hypertension controlled by treatment [4].

The Blood Pressure Control in All Subgroups With Hypertension (BP-CRUSH) study (ClinicalTrials.gov identifier: NCT00791258) evaluated improvement in BP goal achievement after patients who were uncontrolled on prior antihypertensive monotherapy were switched to a fixed-dose of amlodipine/olmesartan medoxomil (AML/OM), with or without hydrochlorothiazide (HCTZ), combination treatment regimen [5]. Fixed-dose combination therapy is one proven strategy to escalate therapy in patients who have hypertension uncontrolled by a single agent alone. In one study of 45 Canadian family practice sites, a simplified approach to hypertension treatment employing fixed-dose combination therapy was compared against treatment as usual, which resulted in significantly improved BP control of $64.7 \%$ versus $52.7 \%$, respectively $(P=0.026)$ [6]. Fixeddose combination therapy also has the added benefit of improving adherence [7] and longterm savings for the healthcare system, despite potentially higher out-of-pocket costs [8].

The purpose of this secondary analysis is to present the BP goal achievement rates, BP reductions, and safety findings of study patients in the primary BP-CRUSH study who did not achieve BP control with prior calcium channel blocker (CCB) or angiotensin II receptor blocker (ARB) monotherapy at baseline, and who were subsequently escalated to AML/OM \pm HCTZ fixed-dose combination therapy.

\section{MATERIALS AND METHODS}

\section{Study Design}

The BP-CRUSH study was a 20-week, prospective, open-label, multicenter, phase 4 (3b in South Africa) study in 999 patients with hypertension. Complete inclusion and exclusion criteria have been published previously [5]. Briefly, patients 18-80 years of age were eligible to enter the study if their mean seated cuff systolic BP (SeSBP) was $\geq 140 \mathrm{mmHg}$ (or $\geq 130 \mathrm{mmHg}$ in patients with diabetes mellitus) and $\leq 180 \mathrm{mmHg}$, and their mean seated cuff diastolic BP (SeDBP) was $\leq 110 \mathrm{mmHg}$ after at least 1 month of antihypertensive monotherapy. Patients uncontrolled on multiple antihypertensive therapies (including fixed-dose combination therapy, except for triamterene/HCTZ); with type 1 or 2 diabetes mellitus requiring insulin; type 2 diabetes mellitus and hemoglobin $\mathrm{A}_{1 \mathrm{c}}\left(\mathrm{HbA}_{1 \mathrm{c}}\right) \geq 9.0 \%$ at screening, and serum creatinine levels $>2.0 \mathrm{mg} / \mathrm{dL}$ or calculated glomerular filtration rate $<40 \mathrm{~mL} / \mathrm{min}$ at screening; significant cardiac disease; or serious systemic diseases or secondary hypertension, 
as well as pregnant or lactating women, were excluded. The cohorts of patients who were uncontrolled on prior monotherapy with a CCB or ARB were included in this secondary analysis. Patients provided signed informed consent before participating in any study procedures. The study protocol, amendment, informed consent forms, and information sheets were approved by the appropriate Independent Ethics Committees or Institutional Review Boards. The study was conducted in compliance with the Declaration of Helsinki and in accordance with the International Conference on Harmonization E6 Guideline for Good Clinical Practice (GCP), and United States Food and Drug Administration GCP guidelines.

Figure 1 shows the BP-CRUSH study design. After eligibility was determined, patients were switched from prior monotherapy to fixed-dose AML/OM 5/20 mg for 4 weeks. Patients were up-titrated to higher doses at 4-week intervals on the following schedule: AML/OM 5/40 mg, AML/OM 10/40 mg, AML/OM 10/40 mg + HCTZ $12.5 \mathrm{mg}$, and AML/OM 10/40 mg + HCTZ $25 \mathrm{mg}$. Up-titration of dose was dependent on mean seated cuff BP (SeBP) measurements taken at treatment visits using an Omron ${ }^{\circledR}$ HEM-705CP automated BP monitor (Omron Corporation, Kyoto-Shi Kyoto, Japan). The mean of three SeBP measurements was used to determine if up-titration was necessary.

Patients were up-titrated to higher dosages of AML/OM if their mean SeSBP was $\geq 120$ and $<200 \mathrm{mmHg}$, or their mean SeDBP was $\geq 70$ and $<115 \mathrm{mmHg}$. Patients were up-titrated to any HCTZ-containing dose if their mean SeSBP was $\geq 125$ and $<200 \mathrm{mmHg}$ and/or mean SeDBP was $\geq 75$ and $<115 \mathrm{mmHg}$. Patients whose BP was controlled at the end of a 4 -week interval (BP $<120 / 70 \mathrm{mmHg}$ for AML/OM doses or $<125 / 75 \mathrm{mmHg}$ for HCTZ-containing doses) remained at the same dose until the end of the study, or until their BP became uncontrolled (systolic BP $[\mathrm{SBP}] \geq 130 \mathrm{mmHg}$ or diastolic BP $[\mathrm{DBP}] \geq 80 \mathrm{mmHg}$ ). If their BP became uncontrolled, patients were up-titrated to the next dosage in the treatment algorithm.

\section{Efficacy Assessments}

The primary efficacy endpoint was the cumulative proportion of patients achieving the SeSBP goal of $<140 \mathrm{mmHg}$ (or $<130 \mathrm{mmHg}$ in patients with diabetes) after 12 weeks of treatment.

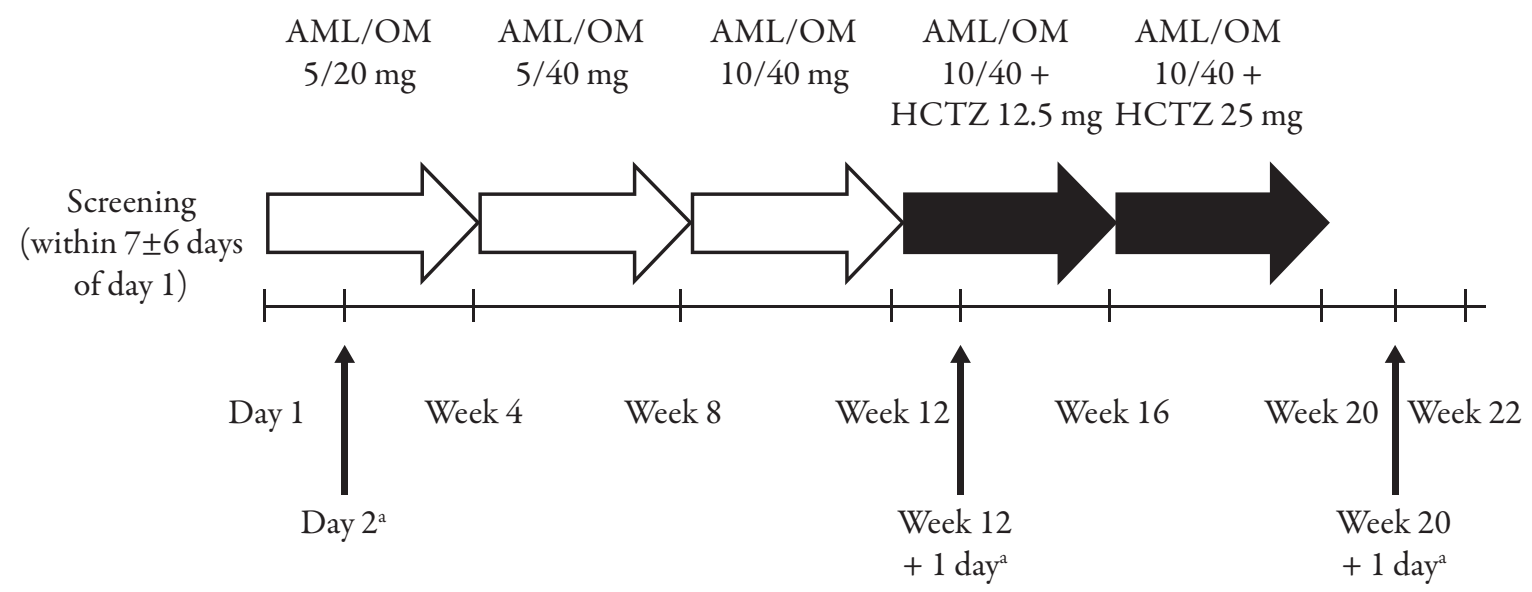

Fig. 1 Study design. $A B P M$ ambulatory blood pressure monitoring, $A M L$ amlodipine, $H C T Z$ hydrochlorothiazide, $O M$ olmesartan medoxomil. a Scheduled ABPM measurement for ABPM cohort 
Secondary efficacy endpoints included a cumulative SeBP goal of $<140 / 90 \mathrm{mmHg}$ (or $<130 / 80 \mathrm{mmHg}$ in patients with diabetes) at weeks 12 and 20, the reduction from baseline (last observation carried forward [LOCF]) in mean SeBP by titration dose, the cumulative SeBP goal of $<140 / 90 \mathrm{mmHg}$ by titration dose, the change from baseline in mean ambulatory BP at weeks 12 and 20 over 24 hours, daytime (8 AM-4 PM), nighttime (10 PM-6 AM), and during the last 2, 4, and 6 hours of the dosing interval, and the proportions of patients achieving mean 24-hour, daytime, and nighttime ambulatory BP targets after 12 and 20 weeks of treatment.

\section{Safety Assessments}

Safety variables measured included adverse events, laboratory parameters, vital signs, and physical examinations. Adverse events were collected and reported from the time of entry into the study for up to 14 days after the last dose of the study drug. An assessment of the severity of adverse events was made, as was a determination of causality with respect to study treatment.

\section{Statistical Analysis}

Demographics and baseline characteristics of the study population were summarized using descriptive statistics. The cumulative BP goal achievement rate was defined as the number of patients achieving BP goal at any time from the first dose date until the end of the period of interest (i.e., specified week or dosage) divided by the total number of patients having a postbaseline BP measurement for the same period of interest. Changes in SeBP and ambulatory BP from baseline were summarized by titration dose (LOCF) and by visit without the LOCF method, and with descriptive statistics, and analyzed by a one-sample paired $t$-test with corresponding standard errors and 95\% CIs. For measurements that employed LOCF, the last post-baseline measurement within a treatment period was carried forward to the end of that same period before being used in the analysis.

\section{RESULTS}

\section{Baseline Demographics}

A total of 999 patients were enrolled into the primary study wherein 118 patients had uncontrolled BP on prior CCB monotherapy and 237 patients had uncontrolled BP on prior ARB monotherapy. Table 1 shows the baseline demographics of the two subgroups of interest. The mean age of patients in this subanalysis was 54.9 years in the prior CCB group and 55.5 years in the prior ARB group, with $20.3 \%$ and $23.6 \%$ of patients aged 65 years or older, respectively. The prior CCB group had more female patients (59.3\%) compared with the prior ARB group (39.2\%). Patients in both cohorts had a body mass index in the obese range of approximately $31 \mathrm{~kg} / \mathrm{m}^{2}$. The majority of patients in the prior ARB group were Caucasian (69.6\%), whereas only $48.3 \%$ were Caucasian in the prior CCB group, with the main difference being that the proportion of Black patients in the prior ARB group was less than one-half of that reported in the prior CCB group (15.2\% vs. $35.6 \%$, respectively). There were more patients with type 2 diabetes and slightly less patients with metabolic syndrome in the prior ARB treatment group compared with the prior CCB group.

Mean ( \pm SD) baseline SeBP was 153.4 $( \pm 9.3) / 91.5( \pm 7.9) \mathrm{mmHg}$ in the prior ССВ group compared with $154.6( \pm 9.2) / 92.6( \pm 8.4)$ $\mathrm{mmHg}$ in the prior ARB group. Mean $( \pm$ SD) 24-hour ambulatory BP was $133.6( \pm 6.9) / 80.7$ $( \pm 7.2) \mathrm{mmHg}$ in the prior CCB group $(n=23)$ 
Table 1 Demographics and baseline characteristics

\begin{tabular}{|c|c|c|}
\hline Characteristic & $\begin{array}{l}\text { Prior CCB } \\
(n=118)\end{array}$ & $\begin{array}{l}\text { Prior ARB } \\
(n=237)\end{array}$ \\
\hline Age, years, mean $( \pm S D)$ & $54.9(11.6)$ & $55.5(11.3)$ \\
\hline Age $\geq 65$ years, $n(\%)$ & $24(20.3)$ & $56(23.6)$ \\
\hline Female, $n(\%)$ & $70(59.3)$ & $93(39.2)$ \\
\hline Weight, kg, mean $( \pm S D)$ & $86.0(17.0)$ & $91.5(22.7)$ \\
\hline Body mass index, $\mathrm{kg} / \mathrm{m}^{2}$, mean $( \pm \mathrm{SD})$ & $30.9(5.3)$ & $31.3(6.3)$ \\
\hline \multicolumn{3}{|l|}{ Race, $n(\%)$} \\
\hline Caucasian & $57(48.3)$ & $165(69.6)$ \\
\hline Black & $42(35.6)$ & $36(15.2)$ \\
\hline Asian & $19(16.1)$ & $34(14.3)$ \\
\hline American Indian/Alaskan Native & $0(0.0)$ & $2(0.8)$ \\
\hline \multicolumn{3}{|l|}{ Ethnicity, $n(\%)$} \\
\hline Hispanic/Latino & $1(0.8)$ & $37(15.6)$ \\
\hline Type 2 diabetes mellitus, $n(\%)$ & $17(14.4)$ & $47(19.8)$ \\
\hline Metabolic syndrome, $n(\%)$ & $57(48.3)$ & $108(45.6)$ \\
\hline Glucose, $\mathrm{mg} / \mathrm{dL}$, mean $( \pm \mathrm{SD})$ & $103.3(18.9)$ & $104.7(21.6)$ \\
\hline High-density lipoprotein, mg/dL, mean $( \pm \mathrm{SD})$ & $51.8(15.6)$ & $53.2(16.8)$ \\
\hline Triglycerides, $\mathrm{mg} / \mathrm{dL}$, mean $( \pm \mathrm{SD})$ & $153.0(89.9)$ & $157.0(93.1)$ \\
\hline $\mathrm{SeBP}, \mathrm{mmHg}$, mean $( \pm \mathrm{SD})$ & $153.4(9.3) / 91.5(7.9)$ & $154.6(9.2) / 92.6(8.4)$ \\
\hline ABPM subgroup, $n$ & 23 & 84 \\
\hline 24-hour ambulatory BP, mmHg, mean $( \pm \mathrm{SD})$ & $133.6(6.9) / 80.7(7.2)$ & $136.2(12.3) / 81.5(9.0)$ \\
\hline \multicolumn{3}{|l|}{ Prior antihypertensive therapy agent, $n(\%)^{a}$} \\
\hline Losartan & - & $43(18.1)$ \\
\hline Valsartan & - & $79(33.3)$ \\
\hline Amlodipine & $93(78.8)$ & - \\
\hline
\end{tabular}

$A B P M$ ambulatory BP monitoring, $A R B$ angiotensin II receptor blocker, $B P$ blood pressure, $C C B$ calcium channel blocker, $S D$ standard deviation, $S e B P$ seated cuff BP

${ }^{a}$ Only ARBs and CCBs for which $\geq 30$ patients had received prior monotherapy with one of these agents are included, as the $n$-values $<30$ were considered to be too small to perform statistical analyses

compared with $136.2( \pm 12.3) / 81.5( \pm 9.0) \mathrm{mmHg}$ in the prior ARB group $(n=84)$. In the prior CCB group, $75.5 \%$ of patients were up-titrated to the maximal dose of AML/OM 10/40 mg + HCTZ $25 \mathrm{mg}$ daily. By comparison, $69.7 \%$ were titrated to the maximal dose in the prior ARB group.

A breakdown of prior antihypertensive therapy in the prior CCB and prior ARB groups by specific drug showed that most patients in the prior CCB group had been previously taking AML monotherapy $(n=93 ; 78.8 \%)$.
The other CCBs used as prior monotherapy included nifedipine, nisoldipine, isradipine, and felodipine. The most widely used ARBs in the prior ARB monotherapy group were valsartan $(n=79 ; 33.3 \%)$ and losartan $(n=43 ; 18.1 \%)$, and statistical analyses were performed for these two ARBs; however, analyses were not performed for the other ARBs used as prior monotherapy, which included telmisartan, irbesartan, and candesartan, because of the small numbers of patients in these subgroups. 


\section{SeBP}

Figure 2 shows the SeSBP goal achievement rates by week 12 for the two subgroups of interest. The majority of patients achieved an SeSBP of $<140 \mathrm{mmHg}$ (or $<130 \mathrm{mmHg}$ in patients with diabetes) in both prior therapy groups, with $4.2 \%$ fewer patients in the prior CCB group achieving this goal compared with the prior ARB group. For comparison, $76.2 \%$ and $77.9 \%$ of patients specifically taking prior ARB monotherapy with losartan or valsartan, respectively, achieved this same SeSBP goal. A cumulative SeBP goal of $<140 / 90 \mathrm{mmHg}$ (or $<130 / 80 \mathrm{mmHg}$ in patients with diabetes) was achieved in $65.8 \%$ and $83.8 \%$ of patients in the prior CCB group by weeks 12 and 20, respectively. In the prior ARB group, cumulative SeBP goal achievement was $72.2 \%$ and $86.8 \%$ by weeks 12 and 20 , respectively.

The proportions of patients achieving the SeBP threshold of $<140 / 90 \mathrm{mmHg}$ in the prior $\mathrm{CCB}$ and prior $\mathrm{ARB}$ subgroups at the highest dual-combination therapy (AML/OM 10/40 mg) and triple-combination therapy (AML/OM

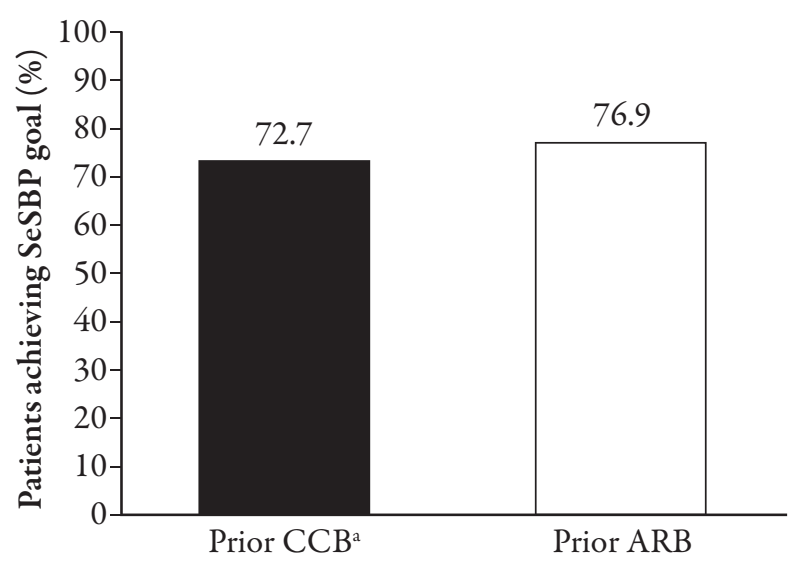

Fig. 2 The proportion of patients achieving the SeSBP goal of $<140 \mathrm{mmHg}$ (or $<130 \mathrm{mmHg}$ for patients with diabetes) in the prior $\mathrm{CCB}$ and prior $\mathrm{ARB}$ subgroups. $A R B$ angiotensin II receptor blocker, $C C B$ calcium channel blocker, $S e S B P$ seated cuff systolic blood pressure. ${ }^{a}$ The majority (80\%) of patients in the prior $\mathrm{CCB}$ subgroup had been taking amlodipine monotherapy
10/40 mg + HCTZ $25 \mathrm{mg}$ ) doses are shown in Figure 3. A greater proportion of patients achieved this threshold in the prior ARB group at both titration doses compared with the prior CCB group.

Figure 4 shows the proportion of patients achieving the SeBP threshold of $<140 / 90 \mathrm{mmHg}$ at the highest dual-combination therapy (AML/ OM 10/40 mg) and triple-combination therapy (AML/OM 10/40 mg + HCTZ $25 \mathrm{mg}$ ) doses for the prior ARB monotherapy losartan $(n=41)$ and valsartan $(n=76)$ subgroups. SeBP goal achievement was similar between prior losartan and valsartan monotherapy subgroups at the AML/OM 10/40 mg dose; however, a greater proportion of patients in the prior losartan group achieved the SeBP goal compared with the prior valsartan subgroup at the AML/OM 10/40 mg + HCTZ 25 mg dose.

Mean SeSBP was significantly reduced from baseline at the week 12 and week 20 visits in the prior CCB (18.3 and $23.4 \mathrm{mmHg}$, respectively; both $P<0.0001)$ and prior ARB $(23.7$ and $28.5 \mathrm{mmHg}$, respectively; both $P<0.0001)$ subgroups. Figure 5 shows the mean $( \pm$ SE) decrease from baseline in SeBP by titration dose (LOCF). At all titration steps, SeBP was significantly reduced from baseline $(P<0.0001)$ in both the prior CCB and prior ARB groups. A mean $( \pm \mathrm{SE})$ change in SeBP (LOCF) of $-17.9( \pm 1.27) /-9.7( \pm 0.75) \mathrm{mmHg}$ from a baseline SeBP of 153.9/91.7 mmHg was observed in the prior CCB group at the end of the AML/OM $10 / 40 \mathrm{mg}$ dose period. The change from baseline in the prior ARB group was $-22.3( \pm 0.92) /-12.3$ $( \pm 0.55) \mathrm{mmHg}$ from a baseline SeBP of $155.4 / 93.3$ mmHg. The mean ( \pm SE) change in SeBP (LOCF) after treatment with the maximally titrated dose of AML/OM 10/40 mg + HCTZ $25 \mathrm{mg}$ was -21.8 $( \pm 1.68) /-11.6( \pm 1.12) \mathrm{mmHg}$ from a baseline SeBP of $154.4 / 92.3 \mathrm{mmHg}$ in the prior CCB group, compared with $-26.2( \pm 1.31) /-15.0( \pm 0.86) \mathrm{mmHg}$ from a baseline SeBP of $155.7 / 94.5 \mathrm{mmHg}$ in the 


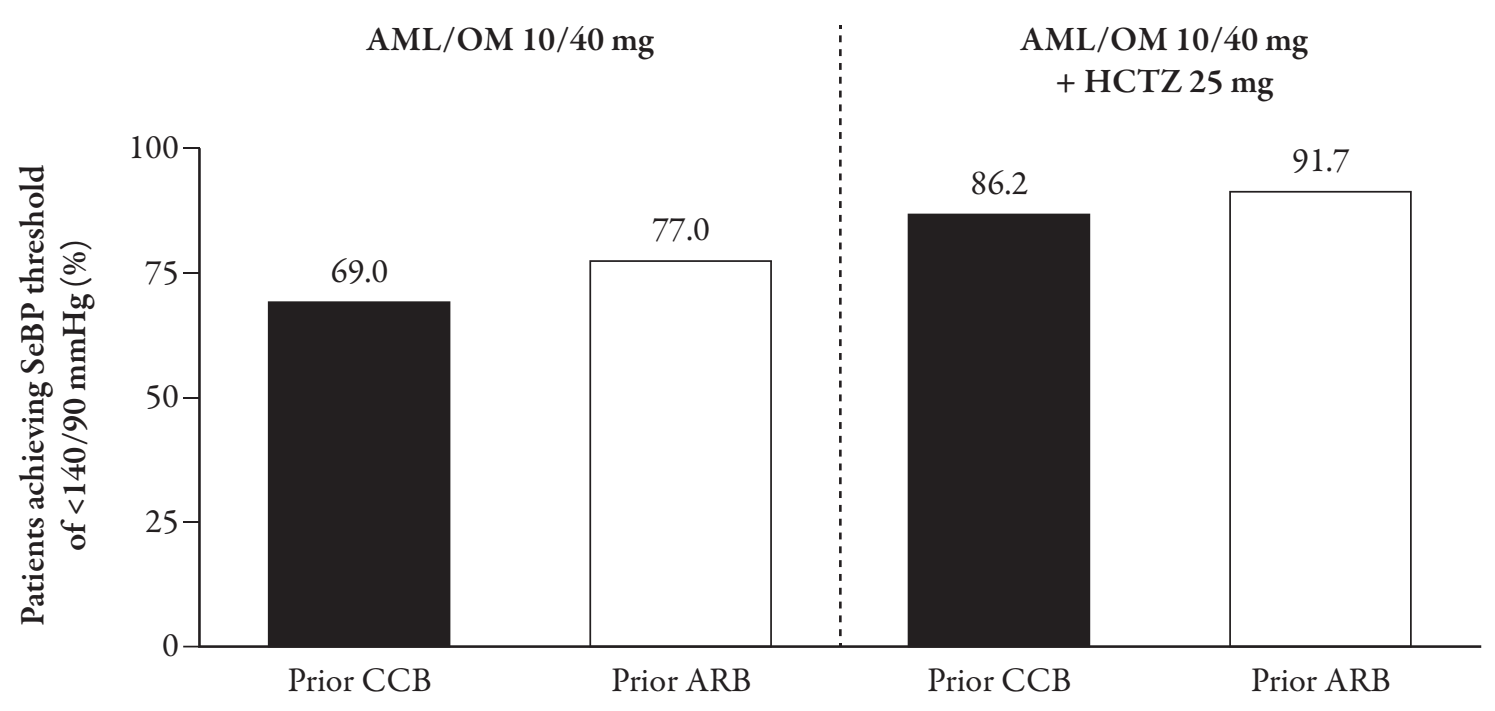

Fig. 3 The proportions of patients achieving the SeBP threshold of $<140 / 90 \mathrm{mmHg}$ in the prior CCB and prior ARB subgroups at the highest dual-combination therapy (AML/OM 10/40 mg) and triple-combination therapy (AML/OM 10/40 mg + HCTZ $25 \mathrm{mg}$ ) doses. $A M L$ amlodipine, $A R B$ angiotensin II receptor blocker, $C C B$ calcium channel blocker, $H C T Z$ hydrochlorothiazide, $O M$ olmesartan medoxomil, SeBP seated cuff blood pressure

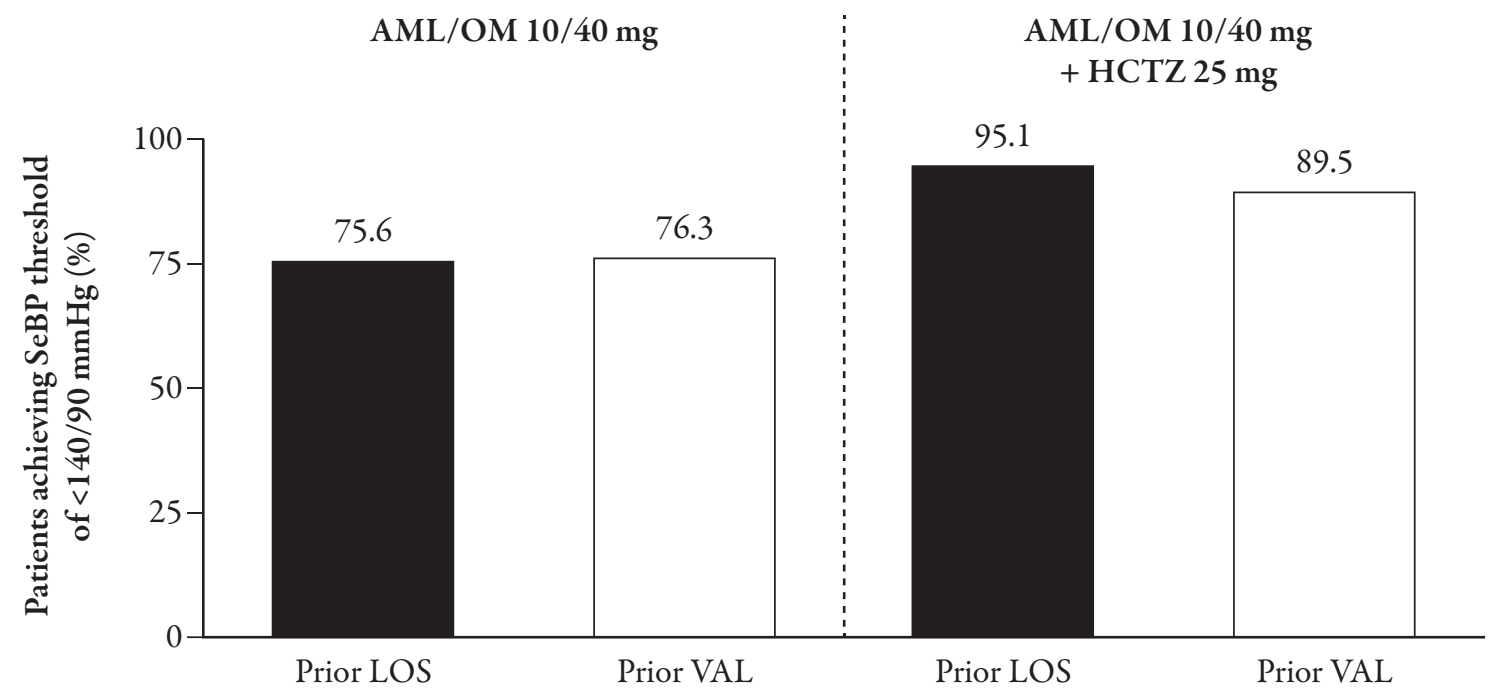

Fig. 4 The proportions of patients achieving the SeBP threshold of $<140 / 90 \mathrm{mmHg}$ in the prior angiotensin II receptor blocker subgroups by agent (LOS and VAL) at the highest dual-combination therapy (AML/OM 10/40 mg) and triplecombination therapy (AML/OM 10/40 mg + HCTZ $25 \mathrm{mg}$ ) doses. AML amlodipine, HCTZ hydrochlorothiazide, LOS losartan, $O M$ olmesartan medoxomil, $S e B P$ seated cuff blood pressure, $V A L$ valsartan

prior ARB group. Results for the prior losartan and valsartan subgroups were similar to those observed for the prior ARB monotherapy group, with SeBP reductions generally being greater overall for losartan compared with valsartan across the $\mathrm{AML} / \mathrm{OM} \pm \mathrm{HCTZ}$ titration regimen.
Mean $( \pm$ SE) SeBP reductions ranged from 18.5 $( \pm 1.9) / 9.5( \pm 1.3) \mathrm{mmHg}$ to $28.3( \pm 3.3) / 18.7$ $( \pm 1.9) \mathrm{mmHg}$ for prior losartan and 13.4 $( \pm 1.4) / 7.4( \pm 0.8) \mathrm{mmHg}$ to $25.2( \pm 2.2) / 13.2$ $( \pm 1.3) \mathrm{mmHg}$ for prior valsartan monotherapy. At all titration steps, SeBP was significantly 

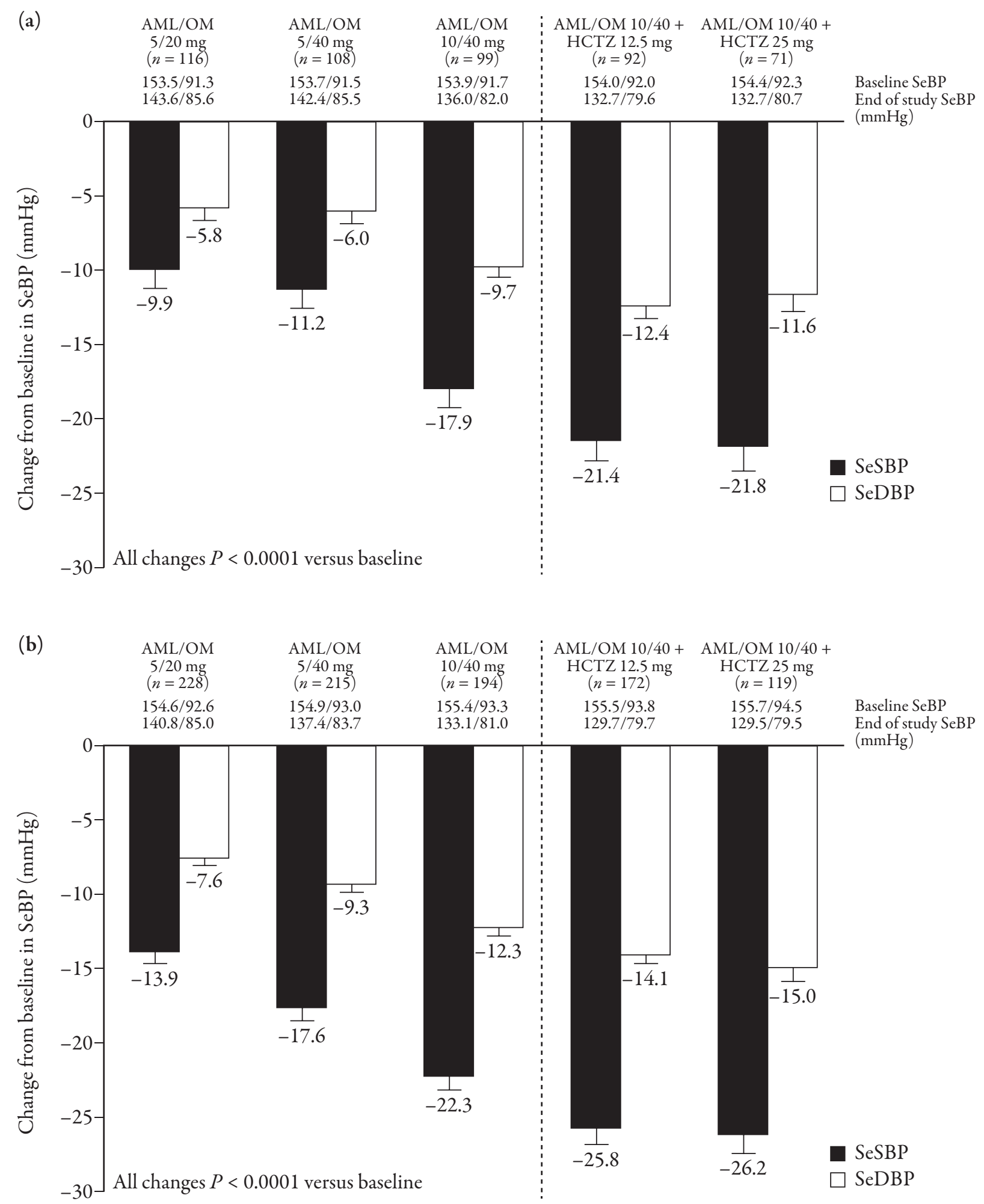

Fig. 5 Change from baseline in mean ( \pm SE) SeBP by titration dose (last observation carried forward) in the (a) prior calcium channel blocker, (b) prior angiotensin II receptor blocker, (c) prior losartan, and (d) prior valsartan subgroups. $A M L$ amlodipine, $H C T Z$ hydrochlorothiazide, $O M$ olmesartan medoxomil, $S e B P$ seated cuff blood pressure, SeDBP seated cuff diastolic blood pressure, SeSBP seated cuff systolic blood pressure 

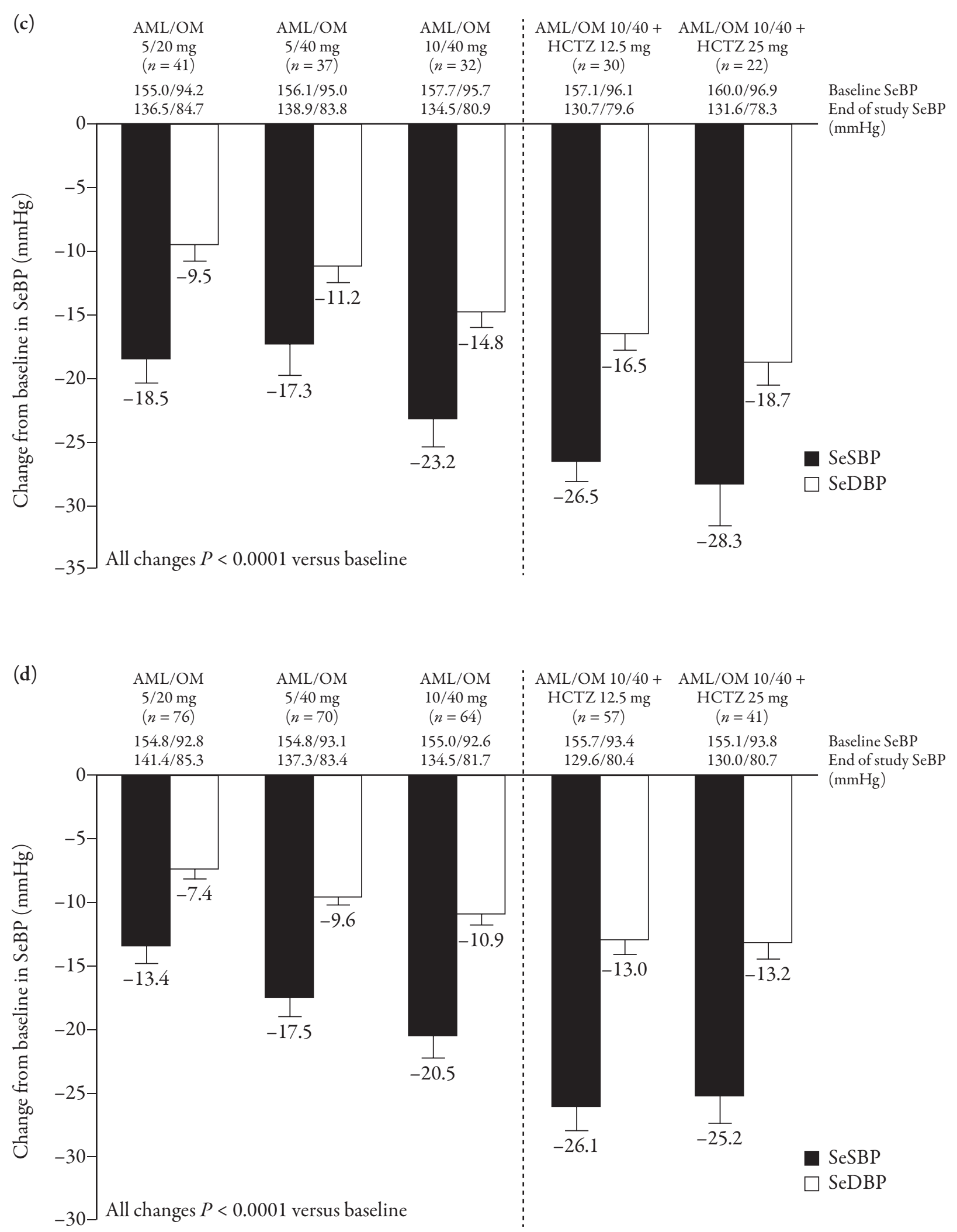

Fig. 5 continued 
reduced from baseline $(P<0.0001)$ in both the prior valsartan and prior losartan groups.

\section{4-Hour Ambulatory BP Monitoring}

Figure 6 shows the change from baseline in mean 24-hour, daytime, and nighttime ambulatory BP through weeks 12 and 20. By week 12 , the mean ( \pm SE) 24-hour change from baseline in ambulatory BP was $-11.1( \pm 1.91) /-6.9( \pm 1.41) \mathrm{mmHg}$ from a baseline BP of $134.1 / 79.8 \mathrm{mmHg}$ in the prior CCB group, compared with -16.4 ( \pm 1.43)/ -10.1 ( \pm 0.85) $\mathrm{mmHg}$ from a baseline BP of $137.4 / 82.8 \mathrm{mmHg}$ in the prior ARB group. At week 20, the mean ( \pm SE) 24-hour change from baseline in ambulatory BP was $-19.9( \pm 2.72) /-13.5( \pm 2.04) \mathrm{mmHg}$ from a baseline BP of $135.3 / 83.0 \mathrm{mmHg}$ in the prior CCB group, compared with $-21.3( \pm 1.63)$ / $-13.2( \pm 0.92) \mathrm{mmHg}$ from a baseline BP of $136.4 / 81.7 \mathrm{mmHg}$ in the prior ARB group.

Figure 7 shows ambulatory BP monitoring (ABPM) target achievement rates by weeks 12 and 20 by subgroup for the 24-hour, daytime, and nighttime ABPM targets of $<130 / 80 \mathrm{mmHg}$, $<135 / 85 \mathrm{mmHg}$, and <120/70 $\mathrm{mmHg}$, respectively. ABPM target achievement rates by week 12 in the prior CCB group were $64.7 \%$, $64.7 \%$, and $47.1 \%$, respectively. By comparison, target achievement rates in the prior ARB group were higher at $81.5 \%, 78.5 \%$, and $60.0 \%$, respectively. Target achievement rates by week 20 in the prior CCB group were 84.6\%, 92.3\%, and $84.6 \%$ compared with $91.5 \%, 88.1 \%$, and $79.7 \%$ in the prior ARB group.

Mean ( \pm SE) changes in ambulatory SBP and DBP in the last 2, 4, and 6 hours of the dosing interval were all significantly decreased from baseline at week 12 in the prior CCB (10.6 [2.88]/5.7 [2.31] mmHg, 11.5 [2.85]/ $6.9[2.14] \mathrm{mmHg}$, and 10.6 [2.70]/6.6 [1.99]
$\mathrm{mmHg}$, respectively; all $P<0.05$ vs. baseline) and prior ARB (16.3 [2.03]/9.4 [1.32] $\mathrm{mmHg}$, $15.0[1.75] / 8.7$ [1.19] $\mathrm{mmHg}$, and 14.8 [1.65]/8.5 [1.11] $\mathrm{mmHg}$, respectively; all $P<$ 0.0001 vs. baseline) treatment groups. Mean ( \pm SE) changes in ambulatory SBP and DBP in the last 2, 4, and 6 hours of the dosing interval were also all significantly decreased from baseline at week 20 in the prior CCB (20.9 [3.14]/14.1 [1.61] mmHg, 19.5 [2.77]/13.0 [2.04] $\mathrm{mmHg}$, and 18.9 [2.69]/13.3 [2.19] $\mathrm{mmHg}$, respectively; all $P<0.0001$ vs. baseline) and prior ARB (20.2 [2.17]/12.3 [1.37] $\mathrm{mmHg}$, $17.4[2.00] / 10.7[1.28] \mathrm{mmHg}$, and 17.4 [1.87]/10.6 [1.21] mmHg, respectively; all $P<0.0001$ vs. baseline). For all ambulatory BP time points at week 12, ambulatory SBP and DBP reductions were greater in prior ARB patients compared with prior CCB patients for the 2-, 4-, and 6-hour time points at week 12 and week 20.

\section{Safety and Tolerability}

Table 2 highlights the incidence of adverse events in the study population. A total of $55.1 \%$ of patients in the prior CCB group experienced a treatment-emergent adverse event (TEAE) compared with $45.6 \%$ of patients in the prior ARB group. The majority of TEAEs were mild-tomoderate in intensity in both subgroups. Serious TEAEs occurred in $0.8 \%$ of patients in the prior CCB group compared with $2.1 \%$ of patients in the prior ARB group. TEAEs judged to be drugrelated occurred in $23.7 \%$ of patients in the prior CCB group compared with $21.1 \%$ of patients in the prior ARB group. TEAEs that led to study discontinuation occurred in $3.4 \%$ of patients in the prior CCB group compared with $6.8 \%$ in the prior ARB group.

Drug-related TEAEs that led to study discontinuation occurred in $3.4 \%$ of patients in the prior CCB group and $5.1 \%$ in the prior 

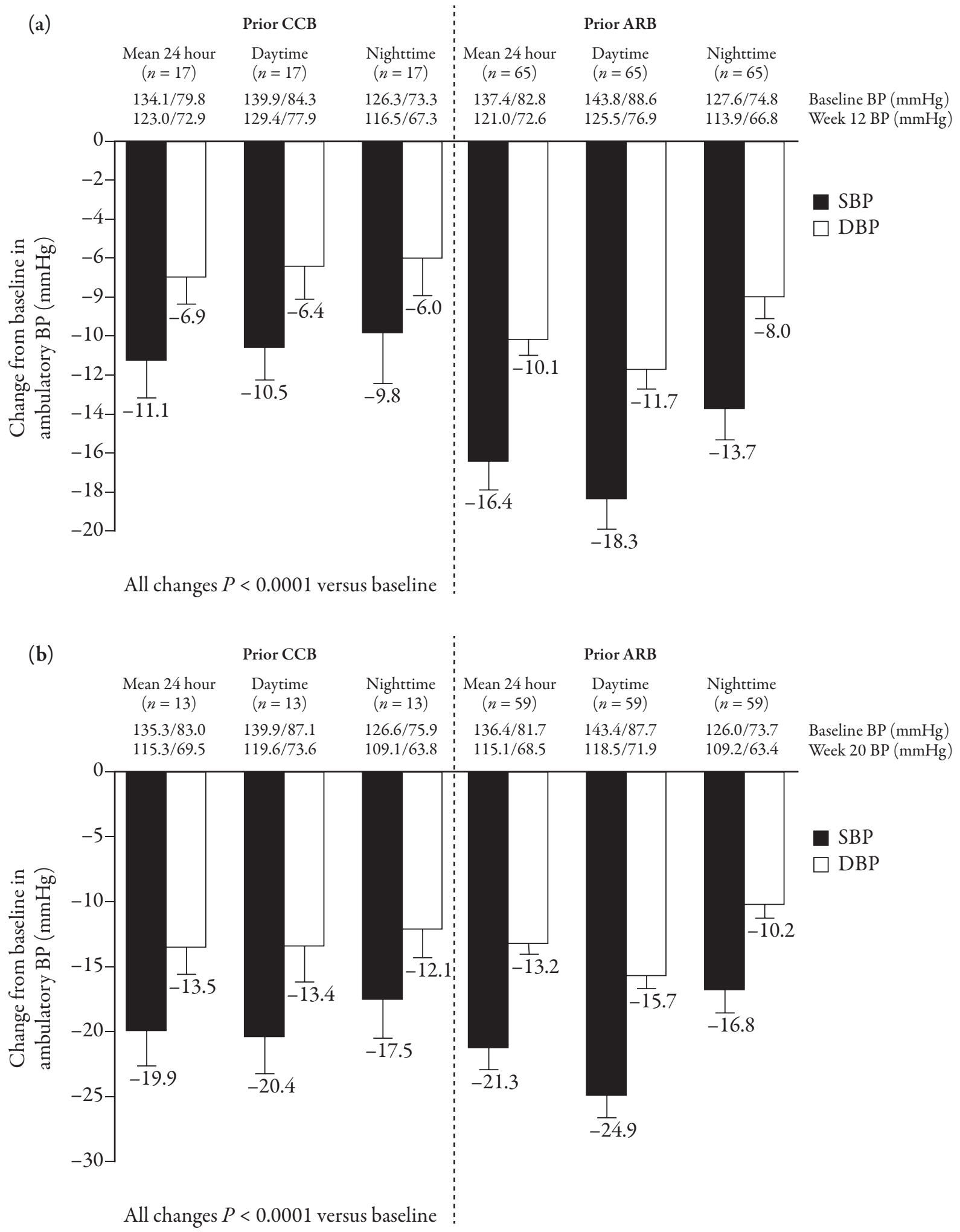

Fig. 6 Change from baseline in the prior $\mathrm{CCB}$ and prior ARB subgroups in mean $( \pm S E)$ 24-hour, daytime, and nighttime ambulatory $\mathrm{BP}$ at (a) week 12 and (b) week $20 . A R B$ angiotensin II receptor blocker, $B P$ blood pressure, $C C B$ calcium channel blocker, $D B P$ diastolic BP, $S B P$ systolic BP 
(a)

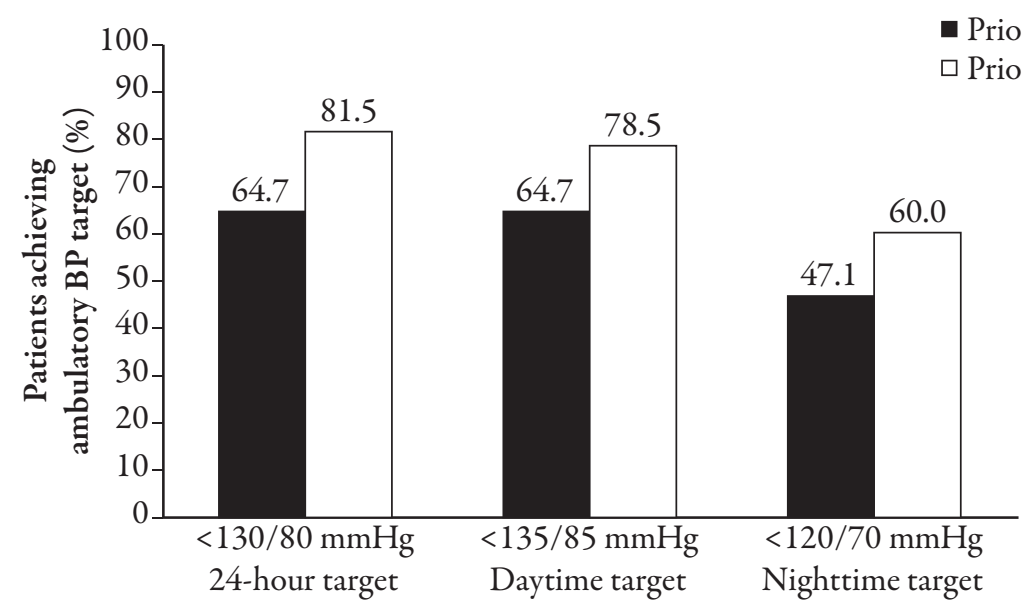

(b)

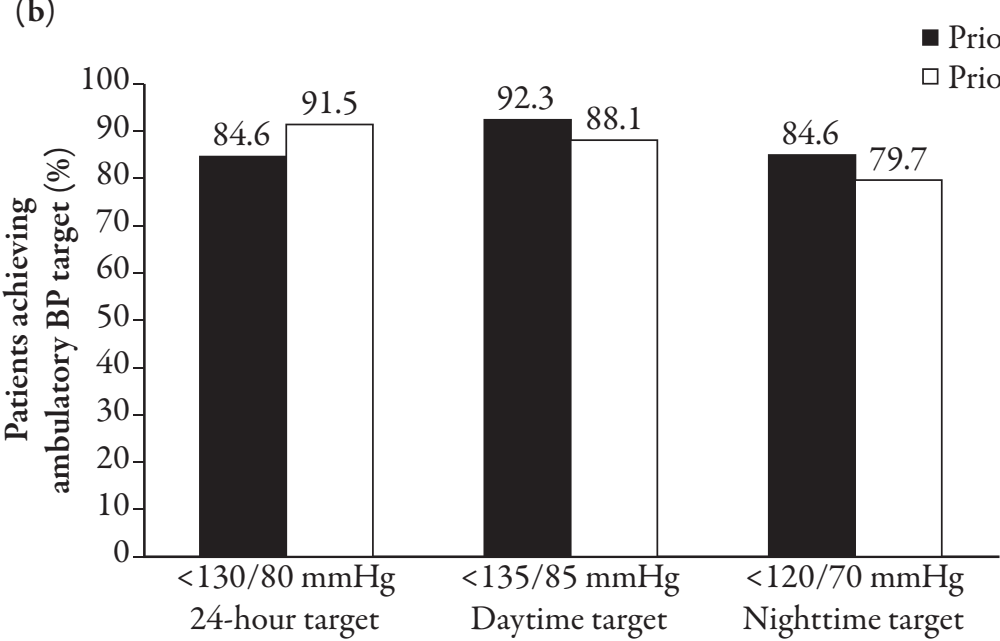

Fig. 7 Mean 24-hour, daytime, and nighttime ambulatory BP target achievement rates in the prior CCB and prior ARB subgroups at (a) week 12 and (b) week 20. $A R B$ angiotensin II receptor blocker, $B P$ blood pressure, $C C B$ calcium channel blocker

ARB group. Table 3 shows the most commonly reported drug-related TEAEs by titration dose and by total for each subgroup. The most frequently reported drug-related TEAEs were dizziness, peripheral edema, hypotension, nausea, fatigue, and muscle spasms. The most frequently reported drug-related TEAE in both the prior CCB and prior ARB groups was peripheral edema at a total rate of $4.2 \%$ and $6.8 \%$, respectively. The highest incidence of drug-related peripheral edema was observed at the maximum AML/OM $10 / 40 \mathrm{mg}$ dose in both the prior CCB (2.0\%) and prior ARB (5.0\%) groups. When HCTZ $12.5 \mathrm{mg}$ and $25 \mathrm{mg}$ were added on to AML/OM, overall rates of drug-related peripheral edema decreased from $3.4 \%$ to $1.1 \%$ and from $5.9 \%$ to $1.1 \%$ in the prior CCB and prior ARB groups, respectively. There were no adverse events leading to death in the BP-CRUSH study.

\section{DISCUSSION}

Uncontrolled hypertension has implications for the patient in terms of increased cardiovascular 
Table 2 Summary of adverse events

\begin{tabular}{lll}
\hline Adverse event, $n(\%)$ & $\begin{array}{l}\text { Prior CCB } \\
(n=118)\end{array}$ & $\begin{array}{l}\text { Prior ARB } \\
(n=237)\end{array}$ \\
\hline TEAEs & $65(55.1)$ & $108(45.6)$ \\
Drug-related TEAEs & $28(23.7)$ & $50(21.1)$ \\
Serious TEAEs & $1(0.8)$ & $5(2.1)$ \\
TEAEs leading to discontinuation & $4(3.4)$ & $16(6.8)$ \\
Most commonly reported drug-related TEAEs \\
Dizziness & $4(3.4)$ & $15(6.3)$ \\
Peripheral edema & $5(4.2)$ & $16(6.8)$ \\
Hypotension & $3(2.5)$ & $3(1.3)$ \\
Nausea & $3(2.5)$ & $1(0.4)$ \\
Fatigue & $1(0.8)$ & $7(3.0)$ \\
Muscle spasms & $3(2.5)$ & $2(0.8)$ \\
\hline
\end{tabular}

$A R B$ angiotensin II receptor blocker, $C C B$ calcium channel blocker, $T E A E$ treatment-emergent adverse event morbidity and mortality, as well as for the healthcare system as a whole with regards to the cost of medical care for patients with hypertension-associated target organ damage. The $\mathrm{AML} / \mathrm{OM} \pm \mathrm{HCTZ}$ titration regimen employed in the primary BP-CRUSH study was an effective means of enabling patients with uncontrolled BP on prior monotherapy with angiotensinconverting enzyme inhibitors or ARBs to reach the primary endpoint of SeSBP $<140 \mathrm{mmHg}$ (or $<130 \mathrm{mmHg}$ in patients with diabetes).

The SeSBP goal at week 12 and SeBP goal achievement at weeks 12 and 20 were consistently higher in the prior ARB group relative to the prior $\mathrm{CCB}$ group. This higher rate of achievement was noted despite the slightly

Table 3 DR-TEAEs by titration dose occurring at an incidence of $\geq 2 \%$

\begin{tabular}{|c|c|c|c|c|c|}
\hline & $\begin{array}{l}\text { AML/OM } \\
5 / 20 \mathrm{mg}\end{array}$ & $\begin{array}{l}\text { AML/OM } \\
5 / 40 \mathrm{mg}\end{array}$ & $\begin{array}{l}\text { AML/OM } \\
10 / 40 \mathrm{mg}\end{array}$ & $\begin{array}{l}\mathrm{AML} / \mathrm{OM} \\
10 / 40 \mathrm{mg}+ \\
\text { HCTZ } 12.5 \mathrm{mg}\end{array}$ & $\begin{array}{l}\text { AML/OM } \\
10 / 40 \mathrm{mg}+ \\
\text { HCTZ } 25 \mathrm{mg}\end{array}$ \\
\hline \multicolumn{6}{|l|}{ Prior CCB group } \\
\hline \multicolumn{6}{|l|}{ Patients exposed to } \\
\hline the dose, $n$ & 118 & 109 & 101 & 94 & 71 \\
\hline \multicolumn{6}{|l|}{ Discontinuation from dose } \\
\hline due to a DR-TEAE, $n(\%)$ & $1(0.8)$ & $0(0.0)$ & $1(1.0)$ & $2(2.1)$ & $0(0.0)$ \\
\hline \multicolumn{6}{|l|}{ TEAE, $n(\%)$} \\
\hline Dizziness & $3(2.5)$ & $0(0.0)$ & $0(0.0)$ & $1(1.1)$ & $0(0.0)$ \\
\hline Peripheral edema & $1(0.8)$ & $1(0.9)$ & $2(2.0)$ & $1(1.1)$ & $0(0.0)$ \\
\hline Nausea & $2(1.7)$ & $0(0.0)$ & $0(0.0)$ & $1(1.1)$ & $0(0.0)$ \\
\hline Syncope & $0(0.0)$ & $0(0.0)$ & $0(0.0)$ & $2(2.1)$ & $0(0.0)$ \\
\hline \multicolumn{6}{|l|}{ Prior ARB group } \\
\hline \multicolumn{6}{|l|}{ Patients exposed to the } \\
\hline dose, $n$ & 237 & 219 & 199 & 175 & 122 \\
\hline \multicolumn{6}{|l|}{ Discontinuation from dose } \\
\hline due to a DR-TEAE, $n(\%)$ & $2(0.8)$ & $2(0.9)$ & $3(1.5)$ & $2(1.1)$ & $3(2.5)$ \\
\hline \multicolumn{6}{|l|}{ TEAE, $n(\%)$} \\
\hline Dizziness & $4(1.7)$ & $2(0.9)$ & $4(2.0)$ & $6(3.4)$ & $2(1.6)$ \\
\hline Peripheral edema & $2(0.8)$ & $2(0.9)$ & $10(5.0)$ & $1(0.6)$ & $1(0.8)$ \\
\hline Hypotension & $0(0.0)$ & $0(0.0)$ & $0(0.0)$ & $0(0.0)$ & $3(2.5)$ \\
\hline
\end{tabular}

$A M L$ amlodipine, $A R B$ angiotensin II receptor blocker, $C C B$ calcium channel blocker, $D R$ drug related, $H C T Z$ hydrochlorothiazide, $O M$ olmesartan medoxomil, TEAE treatment-emergent adverse event 
higher baseline mean SeBP observed in the prior ARB group. The SeSBP goal achievement rate in this substudy was slightly higher in the prior ARB group relative to goal achievement in the total cohort $(n=999)$ of the primary study (76.9\% vs. $75.8 \%$, respectively) and slightly lower in the prior CCB group (72.7\% vs. $75.8 \%)$. CCBs and ARBs have different mechanisms of action and, thus, it might be expected that goal achievement rates would not be identical. A larger study designed to test the efficacy of a regimen that adds on an agent to an existing class of medication versus switching to a different agent when escalating to combination therapy would be required to determine if there is any difference between the two approaches to therapy. It is noteworthy that previously published data from this study demonstrated that $69.3 \%$ and $73.1 \%$ of patients who had BP uncontrolled on prior angiotensin-converting enzyme inhibitor monotherapy achieved SeSBP/ SeDBP and SeSBP goals, respectively, by week 12, and $83.0 \%$ had achieved SeSBP/SeDBP goal by week 20 [5]. These results compare favorably with patients in both the prior $\mathrm{CCB}$ and prior ARB monotherapy groups.

The same higher trend in the prior ARB group relative to the prior CCB group was noted in the change from baseline in mean SeBP and in mean 24-hour ambulatory BP (except for DBP at week 20). The prior ARB group was observed to have a greater reduction in SeBP compared with the prior CCB group by $4.4 / 2.6 \mathrm{mmHg}$ and by $4.4 / 3.4 \mathrm{mmHg}$ after titration to, and treatment with, AML/OM 10/40 mg and AML/ OM 10/40 mg + HCTZ 25 mg, respectively. Compared with the prior CCB group, the prior ARB group had a higher reduction in mean 24-hour ambulatory SBP/DBP by $5.3 / 3.2 \mathrm{mmHg}$ at week 12 , and in mean 24-hour ambulatory SBP of $1.4 \mathrm{mmHg}$ at week 20. Regardless of these differences in BP reduction, patients from both treatment groups had significantly reduced SeBP and ambulatory BP from baseline when switched to an AML/OM regimen, with or without HCTZ.

The achievement of American Heart Association-recommended ambulatory BP targets followed an overall consistent pattern as was observed for the achievement of SeBP goals, and the current titration regimen enabled a majority of patients in both cohorts to achieve these targets. By week 12, the prior ARB group had substantially higher achievement rates for the mean 24-hour BP target of $<130 / 80 \mathrm{mmHg}$, mean daytime target of $<135 / 85 \mathrm{mmHg}$, and mean nighttime target of $<120 / 70 \mathrm{mmHg}$ compared with the prior CCB group. However, at week 20, the prior ARB group had a higher rate of achievement for the mean 24-hour BP target, whereas the prior CCB group had higher rates of achievement for both the daytime and nighttime BP targets.

The AML/OM titration regimen was well tolerated overall in both subgroups. The incidence of TEAEs and drug-related TEAEs were higher in the prior CCB group compared with the prior ARB group, whereas the incidence of drug-related TEAEs leading to discontinuation were lower in the prior CCB group compared with the prior ARB group. Rates of peripheral edema were highest in the prior $\mathrm{CCB}$ and $\mathrm{ARB}$ groups at a dose of AML/OM 10/40 mg daily. The addition of HCTZ $12.5 \mathrm{mg}$ and $25 \mathrm{mg}$ to AML/ $\mathrm{OM}$ decreased the incidence of peripheral edema in both the prior CCB and prior ARB groups. These findings provide additional support to previous observations that the addition of a thiazide diuretic to a regimen containing highdose AML can help to mitigate the incidence of edema $[5,9]$.

A limitation of this study is its open-label, single-arm design, which could potentially introduce treatment bias due to lack of blinding. Another limitation is that patients who volunteer 
to participate in clinical studies are likely to have better adherence to treatment than patients in the general population, potentially increasing the BP goal achievement rates compared with those observed in clinical practice. The strengths of the BP-CRUSH study include the large study population, the utilization of ABPM to assess 24-hour BP control, and the use of aggressive BP criteria for dose titration.

In conclusion, an AML/OM-based titration regimen enabled the achievement of BP control in patients not achieving guidelinerecommended goals with prior CCB or ARB monotherapy. The switching of patients with uncontrolled BP on prior CCB or ARB monotherapy to a fixed-dose titration regimen of AML/OM, with or without HCTZ, did not impact the achievement of the primary outcome in this substudy. A large proportion of patients in both subgroups achieved SeBP goals regardless of baseline monotherapy, and these high rates of BP control were associated with significant reductions in both SeBP and ambulatory BP. Data from this study demonstrate that the treat-to-target BP approach employed in the BP-CRUSH study could be potentially beneficial in overcoming clinical inertia in daily practice. Simple changes to treatment in the clinical environment, such as mandatory up-titration and the addition of other antihypertensive agents if BP goals are not achieved, can translate into real-world benefits in overcoming clinical inertia and improving BP control.

\section{ACKNOWLEDGMENTS}

This study was supported by Daiichi Sankyo, Inc. Medical writing and editorial services were provided by Robert Schupp, PharmD, and Alan J. Klopp, PhD, of inScience Communications, Springer Healthcare. Support for this assistance was funded by Daiichi Sankyo, Inc. Dr. Neutel is the guarantor for this article, and takes responsibility for the integrity of the work as a whole.

Conflict of Interest. Joel Neutel serves as a consultant for Novartis and has received payment for lectures, including services on the speaker's bureau for Takeda, the Bristol-Myers Squibb/Sanofi Pharmaceutical Partnership, Novartis, Pfizer Inc., Boehringer Ingelheim, Forest Laboratories, Merck, and Daiichi Sankyo, Inc. Ali Shojaee and Jen-Fue Maa are employees of Daiichi Sankyo, Inc.

Open Access. This article is distributed under the terms of the Creative Commons Attribution Noncommercial License which permits any noncommercial use, distribution, and reproduction in any medium, provided the original author(s) and source are credited.

\section{REFERENCES}

1. Lewington S, Clarke R, Qizilbash N, Peto R, Collins R; Prospective Studies Collaboration. Age-specific relevance of usual blood pressure to vascular mortality: a meta-analysis of individual data for one million adults in 61 prospective studies. Lancet. 2002;360:1903-13.

2. ALLHAT Officers and Coordinators for the ALLHAT Collaborative Research Group. Major outcomes in high-risk hypertensive patients randomized to angiotensin-converting enzyme inhibitor or calcium channel blocker vs diuretic: The Antihypertensive and Lipid-Lowering Treatment to Prevent Heart Attack Trial (ALLHAT). JAMA. 2002;288:2981-97.

3. Tedesco MA, Natale F, Calabro R. Effects of monotherapy and combination therapy on blood pressure control and target organ damage: a randomized prospective intervention study in a large population of hypertensive patients. J Clin Hypertens (Greenwich). 2006;8:634-41.

4. Yoon SS, Ostchega Y, Louis T. Recent trends in the prevalence of high blood pressure and its treatment and control, 1999-2008. NCHS Data Brief. 2010:1-8.

5. Weir MR, Hsueh WA, Nesbitt SD, et al. A titrateto-goal study of switching patients uncontrolled on antihypertensive monotherapy to fixed-dose combinations of amlodipine and olmesartan medoxomil \pm hydrochlorothiazide. J Clin Hypertens (Greenwich). 2011;13:404-12. 
6. Feldman RD, Zou GY, Vandervoort MK, Wong CJ, Nelson SA, Feagan BG. A simplified approach to the treatment of uncomplicated hypertension: a cluster randomized, controlled trial. Hypertension. 2009;53:646-53.

7. Taylor AA, Shoheiber O. Adherence to antihypertensive therapy with fixed-dose amlodipine besylate/benazepril $\mathrm{HCl}$ versus comparable component-based therapy. Congest Heart Fail. 2003;9:324-32.
8. Rabbani A, Alexander GC. Out-of-pocket and total costs of fixed-dose combination antihypertensives and their components. Am J Hypertens. 2008;21:509-13.

9. Chrysant SG, Melino M, Karki S, Lee J, Heyrman R. The combination of olmesartan medoxomil and amlodipine besylate in controlling high blood pressure: $\mathrm{COACH}$, a randomized, double-blind, placebo-controlled, 8-week factorial efficacy and safety study. Clin Ther. 2008;30:587-604. 